UNIVERSIDADE DE SÃO PAULO

INSTITUTO DE FÍSICA E QUIMMICA DE SÃO CARLOS

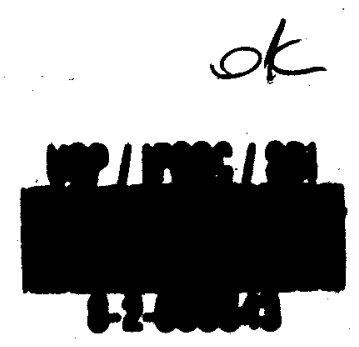

\title{
O POTENCIAL VAN DER WAALS DIPOLO-QUADRUPOLO
}

\author{
JOSÉ CARLOS ANTÔNIO
}

Tese apresentada ao Instituto de Física o Química de São Carlos, USP, para obtenção do título de Mestre em Física.

Orientador: Silvestre Ragusa

DEPAARTAMENTO DE FÍSICA E CIENNCIA DOS MATERIAIS

São Carlos - 1981 

Ao meu pai e à minha mãe 


\section{AGRADECIMENTOS}

Ao Prof. Silvestre Ragusa pelo seu interesse, ensina mentos, dedicação e paciência na orientação deste tratablho.

Ao Prof. György Csanak que sugeriu o problema e me orientou no inicio deste trabalho.

Aos Profs. Josē Lindoso, Octāvio Hamilton Botelho Mou rão, Roberto Vieira, Raiumundo Moacir Lima Filho, Heyrton Bessa, Guillermo Cabrera, Antônio Penna, Roland Kuberle pelos estimulos e amizade.

Aos amigos Emerson Pires Leal e Luiz Eugênio Machado pelos contatos e amizade.

Ao Departamento de Física do Instituto de Física $e^{* *}$ Química de são carlos pela atenção que sempre me foi dada.

A Universidade do Amazonas e ao P.I.C.D pelo apoio Einanceiro. 


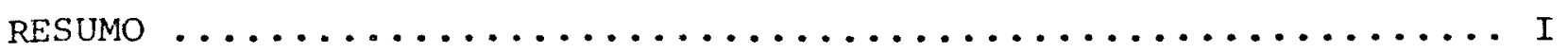

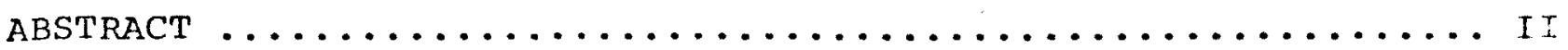

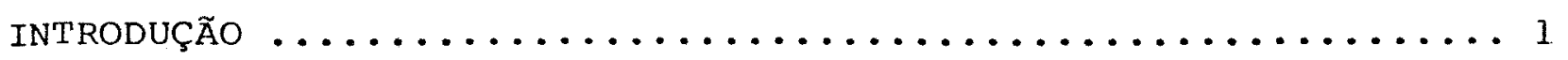

CAPITULO I - A INTERAÇÃO DE VAN DER WAALS DIPOLO-QUADRUPOLO INDUZ IDO ENTRE DOIS ATOMOS DE HIDROGENIO $\ldots \ldots \ldots 3$

CAPITULO II - AS FORÇAS DE DISPERSÕES DE LONDON NA INTERAÇÃO

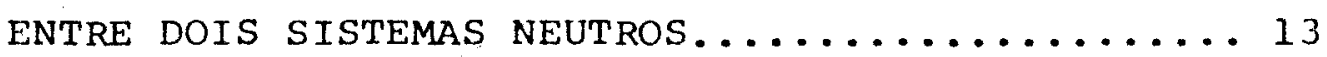

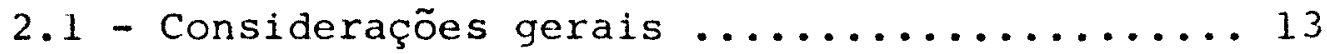

2.2 - A interação dipolo-dipolo das forças de dispersões de London $\ldots \ldots \ldots \ldots \ldots \ldots \ldots 21$

CAP ITULO III - A INTERAÇAOO DIPOLO-QUADRUPOLO DAS FORÇAS DIS

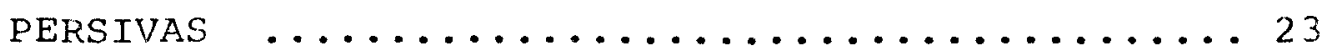

CONTRIBUIÇŐS ORIGINAIS DESTE TRABALHO $\ldots \ldots \ldots \ldots \ldots \ldots \ldots \ldots$

CONCLUSÃO $\ldots \ldots \ldots \ldots \ldots \ldots \ldots \ldots \ldots \ldots \ldots \ldots \ldots \ldots \ldots$

APENDICE I - A POLARIZABILIDADE $\ldots \ldots \ldots \ldots \ldots \ldots \ldots \ldots \ldots \ldots \ldots \ldots \ldots \ldots \ldots \ldots$

REFERENCIAS BIBLIOGRAFICAS $\ldots \ldots \ldots \ldots \ldots \ldots \ldots \ldots \ldots \ldots \ldots \ldots \ldots \ldots \ldots$ 
RESUMO

Estudamos a parte dipolo-quadrupolo da energia de interação entre dois sistemas neutros, para distâncias intermediárias e grandes em relação às dimensões lineares dos mesmos. 


\section{ABSTRACT}

We study the dipole-quadrupole part of the interaction energy between two neutral systems for intermediary distances and large distances in relation to its linears dimensions. 
INTRODUÇÃO

Neste trabalho estudamos a energia de interação dipo10-quadrupolo entre dois sistemas neutros separados entre si por uma distância intermediāria e grande em relação a soma das dimen soes lineares dos sistemas.

Como preparação ao estudo da situação geral considera mos no capitulo I a situação particular na qual os sistemas são dois átomos de hidrogênio e focalizamos o caso de grandes distân cias entre os núcleos. Fazemos aqui o cālculo para a interação dipolo-quadrupolo estendendo deste modo o cālculo feito por Lon Ion [9] para a parte dipolo-dipolo da interação. A expressão per turbada da energia de interaçäo do sistema é estabelecida e atra vés desta expressäo calculamos um limite superior para a energia. Um limite inferior pode ser conseguido empregando o método variacional e o determinamos logo em seguida. Na parte final do capitulo mostramos como a correção da energia dipolo-quaárupolo pode ser relacionada com as polarizabilidades elétrica dependente da frequëncia. Isto é feito de maneira análoga com o que Dalgarno [18] fez para a parte dipolo-dipolo. Aliás, esta dependência é a razão pela qual as forças de Van der Waals são também chamadas de forças de dispersào de London.

No capitulo II consideramos a situação mais geral de dois sistemas atômicos separados por uma distância intermediária e grande (em relação à soma das dimensões lineares dos dois sistemas). Neste capitulo apresentamos as fōrmulas básicas que foram deduzidas por Longuet-Higgins [10] e Boehm e Yaris [1] ]. De 
lineamos aqui o estudo feito por Jacobi e Csanak [16] delineando o cálculo por eles fejto para a interaçao dipolo-dipolo das forças de dispersões de London.

No capitulo III estendenos os resultados do capitulo II. Calculamos a energia de interação dipolo-quadrupolo entre os dois sistemas neutros para distâncias intermediárias e grandes. Mostramos aqui que para $R$ grande recuperamos o resultado apresentado no capítulo I. Na parte final do capitulo mencionamos a importância destes cälculos e onde eles são aplicados. 


\section{CAPITULO I}

A INTERAÇÃO DE VAN DER WAALS DIPOLO-QUADRUPOLO INDUZIDO ENTRE DOIS ATOMOS DE HIDROGENIO

Neste capitulo calcularemos a interação de Var der Waals dipolo-quadrupolo induzido entre dois ātomos de hidrogènio no seu estado fundamental

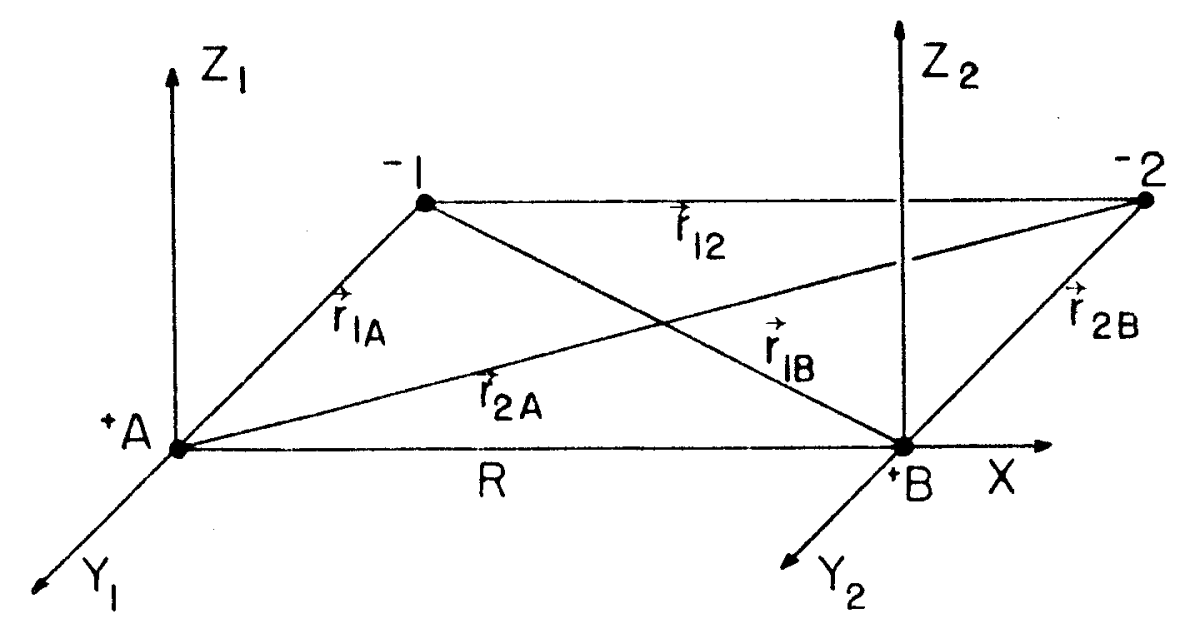

$\mathrm{Na}$ figura acima colocamos as cargas positivas em $\mathrm{A}$ e $B$ e as cargas negativas em 1 e 2 . Os vetores deslocamentos entre as cargas estão nela desenhados. O Hamiltoniano para os dois elétrons é

$\mathrm{H}=\mathrm{H}_{\mathrm{O}}+\mathrm{H}^{\prime}$ 
$H_{O}=-\frac{h^{2}}{2 m}\left(\nabla_{1}^{2}+v_{2}^{2}\right)-\frac{e^{2}}{r_{1 A}}-\frac{e^{2}}{r_{2 B}}$

$$
H^{\prime}=\frac{e^{2}}{R}+\frac{e^{2}}{r_{12}}-\frac{e^{2}}{r_{1 B}}-\frac{e^{2}}{r_{2 B}}
$$

O Hamiltoniano não perturbado $\mathrm{H}_{\mathrm{O}}$ tem a seguinte solução para os dois àtomos de hidrogênio no estado fundamental

$U_{0}\left(\vec{r}_{1}, \vec{r}_{2}\right)=U_{100}\left(\vec{r}_{1}\right) U_{100}\left(\vec{r}_{2}\right)$

Consideraremos a situação na qual $\vec{R}$ é muito grande comparado com $\vec{r}_{1 A}$ e $\vec{r}_{2 A}$. Neste caso $H^{\prime}$ è o têrmo de perturbação. Expandimos agora $H^{\prime}$ em têrmos de $1 / R$, segurando apenas os primeiros termos da expansão. Obtemos desse modo

$$
\begin{aligned}
H^{\prime}= & -\frac{e^{2}}{R^{3}}\left(2 x_{1} x_{2}-y_{1} y_{2}-z_{1} z_{2}\right)+ \\
& +\frac{3 e^{2}}{2 R^{4}}\left[r_{1}^{2} x_{2}-x_{1} r_{2}^{2}+\left(2 y_{1} y_{2}+2 z_{1} z_{2}-3 x_{1} x_{2}\right)\left(x_{1}-x_{2}\right]+\ldots\right.
\end{aligned}
$$

O primeiro tērmo da Eq. (5) representa a energia de" interação dipolo-dipolo. O segundo têrmo da Eq. (5) representa a energia de interação dipolo-quadrupolo.

o valor esperado dos dois têrmos da Eq. (5) entre o es tado $U_{0}\left(\vec{r}_{1}, \vec{r}_{2}\right)$ é zero. Isto porque $U_{0}$ é uma função par de cada componente de $\vec{r}_{1}$ e $\vec{r}_{2}$ separadamente e $H^{\cdot}$ é uma função impar de $\vec{r}_{1}$ e $\vec{r}_{2}$ separadamente. Por isto a energia de interação de van der Waals em primeira ordem de perturbação é zero tanto para a parte dipola-dipolo eomo para a parte dipolo-quadrupolo.

ö tếrmo principal da energia de interação de van der 
Waals è o têrmo em segunda ordem de perturbação, o qual é proporcional a $\left\langle\mathrm{H}^{\prime}\right\rangle\left\langle\mathrm{H}^{\prime}\right\rangle$. Concluimos então que a correção de energia de vido a parte dipolo-dipolo de interação é proporcional a $1 / R^{6}$ (Lon don) e que a correçäo de energia devido a parte dipolo-quadrupolo de interação é proporcional a $1 / R^{\theta}$. Notemos que existe um têrmo de cruzamento envolvendo as duas partes de $H^{\prime}$. Tal têrmo è da ordem de $1 / R^{7}$.

A energia de interação dos dois átomos de hidrogênio em segunda ordem de perturbação é dada pela expressão

$W(R)=\sum_{n}^{\prime} \frac{\left.|<O| H^{\prime}|n\rangle\right|^{2}}{E_{0}-E_{n}}$

A linha no símbolo da soma indica que ela é vālida pa ra todo $n \neq 0$. $E_{o} e ́$ a energia do estado fundamental e $E_{n} \bar{e}$ a energia do estado excitado.

Explicitando os nümeros quānticos de cada sistema em separado escrevemos

$-W(R)=\sum_{\mathrm{n}_{1}, \mathrm{n}_{2}}^{\prime} \frac{\left|<o_{1} \cdot o_{2}\right| \mathrm{H}^{\prime}\left|\mathrm{n}_{1} \cdot \mathrm{n}_{2}\right|^{2}}{\left(\mathrm{E}_{\mathrm{n}_{1}}-\mathrm{E}_{\mathrm{o}_{1}}\right)+\left(\mathrm{E}_{\mathrm{n}_{2}}-\mathrm{E}_{\mathrm{o}_{2}}\right)}$

$\circ_{1} \cdot \circ_{2}$ indicamos números quânticos dos estados fundą mentais $e n_{1} \cdot n_{2}$ indicamos nümeros quänticos de um estado excitado.

Na Eq. (6) $E_{0}<E_{n}$ e o numerador de cáda têrmo da Eq. (6) é positivo, portanto $W(R)$ é negativo. Concluimos então que a energia de interaçäo è atrativa.

Da Eq. (6) é possivel determinar um limite inferior pa 
ra $-W(R)>0$.

Para obter o limite superior substituimos na Eq.

cada $E_{n}$ pela energia $E_{n}$ * do primeiro estado excitado. Obtemos des te modo

$-W(R) \leq \sum_{n}^{\prime} \frac{\left|\left\langle 0\left|H^{\prime}\right| n\right\rangle\right|^{2}}{E_{n *}-E_{0}}$

O denominador da $\mathrm{Eq} \cdot(8)$ pode ser colocado para fora da soma e o numerador pode ser calculado do seguinte modo

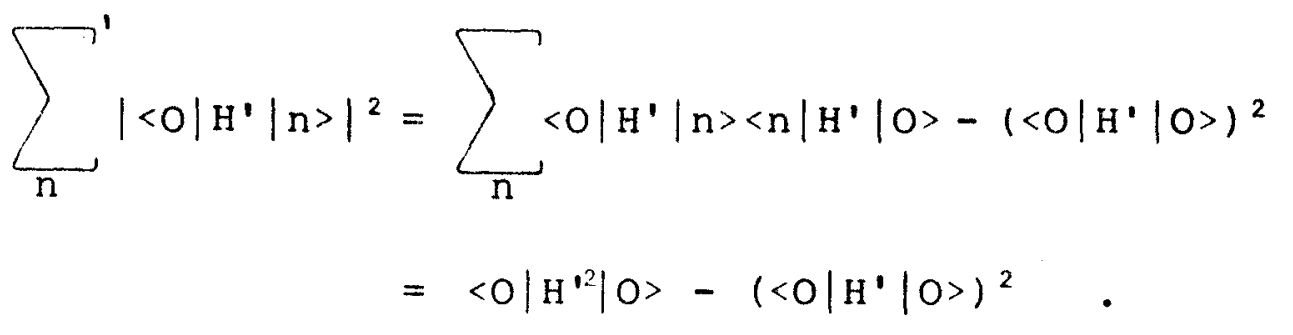

Jà vimos que $\left\langle O\left|H^{\prime}\right| O\right\rangle=0$. Substituindo a L'q. (9)

na Eq. (8) teremos a expressão

$-W(R) \leq \frac{\left\langle O\left|H^{\prime 2}\right| O\right\rangle}{E_{n *}-E_{O}}$.

$\mathrm{Na}$ interação dipolo-dipolo o limite superior da energia de interação é obtido substituindo-se o primeiro tērmo de $H$ ' da Eq. (5) na Eq. (10). O cálculo è conhecido e não o reproduzire mos aqui. Estamos interessados na parte dipolo-quadrupolo da inte raçäo.

Vamos calcular o limite superior da energia de intera Çäo dipolo-quadrupolo. Notemos antes que o têrmo de cruzamento de $\mathrm{H}^{\prime 2}$ não contribui na Eq. (10) pois este têrmo de cruzamento è Im- 
par em pelo menos uma das coordenadas.

$\mathrm{Na}$ interaço dipolo-quadrupolo o limite superior da energia de interaçäo obtido substituindo-se o segundo têrmo de $\mathrm{H}^{\prime}$ da Eq. (5) na Eq. (10) e obtém-se

$$
-W(R) \leq \frac{9 e^{4}}{4 R^{8}} \cdot \frac{\left\langle 0\left|\left[r_{1}^{2} x_{2}-r_{2}^{2} x_{1}+\left(2 y_{1} y_{2}+2 z_{1} z_{2}-3 x_{1} x_{2}\right)\left(x_{1}-x_{2}\right)\right]^{2}\right| 0\right\rangle}{E_{n *}-E_{0}} .
$$

Nesta expressão os àtomos são excitados para estados de número quântico principal 2 de modo que $E_{0}=-2\left(e^{2} / 2 a_{0}\right)$, $E_{n *}=-2\left(e^{2} / 8 a_{0}\right)$ e $E_{n *}-E_{0}=3 e^{2} / 4 a_{0}$.

No cálculo do numerador da Eq. (11) basta tomar os têr mos quadrāticos em cada componente das coordenadas, como por exem plo $r_{1}^{4} x_{2}^{2}$, pois os têrmos cruzados, como exemplo $r_{1}^{2} r_{2}^{2} x_{2} x_{1}$, não dão contribuição. Isto è devido ao fato de que $U_{0}$ é uma função par de cada componente de $\vec{r}_{1}$ e $\vec{r}_{2}$ separadamente e os tērmos cruza dos da Eq. (11) são funções impares de $\vec{r}_{1}$ e $\vec{r}_{2}$ separadamente.

Os cālculos do denominador da Eq. (11) envolvem integrais do tipo

$\int_{0}^{\infty} r^{n} e^{-\alpha r} d r=n ! / \alpha+1$

Calculando o numerador da ti. (11) e substituindo $\circ$ valor $\left(E_{n *}-E_{0}\right)=3 e^{2} / 4 a_{0}$ obtemos

$-W(R) \leq 180 \frac{e^{2} a_{O}^{7}}{R^{8}}$ 
L. Pauling no seu livro $[20]$.

E possivel determinar o limite inferior da energia de interação utilizando o método variacional. A função teste é es colhida do seguinte modo

$\psi\left(\overrightarrow{\mathrm{r}}_{1}, \overrightarrow{\mathrm{r}}_{2}\right)=\mathrm{U}_{100}\left(\overrightarrow{\mathrm{r}}_{1}\right) \mathrm{U}_{100}\left(\overrightarrow{\mathrm{r}}_{2}\right)\left(1+\mathrm{AH}^{\prime}\right)$

Esta escolha foi feita de modo que $\psi\left(\vec{r}_{1}, \vec{r}_{2}\right)$ tem um têrmo proporcional a $H^{\prime}$. Calculando-se o valor esperado de $H^{\prime}$ com $\psi\left(\vec{r}_{1}, \vec{r}_{2}\right)$ aparecerão têrmos proporcional a H'2 . Na Eq. (14) "A" è um parâmetro. A função $\psi\left(\vec{r}_{1}, \vec{r}_{2}\right)$ não é normalizada e determinamos - limite inferior da energia de interaçāo por

$$
E_{O}+W(R) \leq \frac{\iint U_{O}\left(1+A H^{\prime}\right)\left(H_{O}+H^{\prime}\right) U_{O}\left(1+A H^{\prime}\right) d_{r_{1}}^{3} d_{r_{2}}^{3}}{\iint U_{O}^{2}\left(1+A H^{\prime}\right)^{2} d_{r_{1}}^{3} d_{r_{2}}^{3}} \text {. }
$$

O valor esperado dos têrmos de $\mathrm{H}^{\prime}$ e $\mathrm{H}^{\cdot 3}$ entre o estado $\mathrm{U}_{\mathrm{o}}$ é zero. O lado direito da $\mathrm{Eq}$. (15) se reduz a

$$
\begin{aligned}
& \frac{E_{O}+2 A\left\langle O\left|H^{\prime 2}\right| O\right\rangle+A^{2}\left\langle O\left|H^{\prime} H_{O} H^{\prime}\right| O\right\rangle}{\left(1+A^{2}\left\langle O\left|H^{\prime 2}\right| O>\right)\right.} \\
& \quad \text { o valor esperado do têrmo } H^{\prime} H_{O} H^{\prime} \text { entre o estado } U_{O}
\end{aligned}
$$
zero. A Eq. (16) se reduz a

$$
\frac{E_{O}+2 A\left\langle O\left|H^{\prime 2}\right| O\right\rangle}{\left(1+A^{2}\left\langle O\left|H^{\prime 2}\right| O\right\rangle\right)} .
$$

Expandindo o denominador da Eq. (17) e utilizando des- 
ta expansão apenas têrmos da ordem de $\mathrm{H}^{\prime 2}$, que são os têrmos do nosso interesse, obtemos

$$
\frac{\left.\left(E_{O}+2 A<0\left|H^{\prime 2}\right| O\right\rangle\right)}{\left(1+A^{2}\left\langle 0\left|H^{\prime 2}\right| O\right\rangle\right)} \simeq E_{0}+\left(2 A-E_{O} A^{2}\right)\left\langle O\left|H^{\prime 2}\right| O\right\rangle
$$

A Eq. (18) tem um minimo em relação ao parāmetro "A" quando $A=1 / E_{0}$. Relembrando que $E_{0}$ é negativo e substituindo $A=1 / E_{0}$ na Eq. (18) obtemos o limite inferior da energia de interação

$$
E_{O}+W(R) \leq E_{O}+\frac{\left\langle O\left|H^{\prime 2}\right| O>\right.}{E_{O}}
$$

$\mathrm{Na}$ interaçäo dipolo-dipolo o limite inferior da energia de interação pode ser calculado de maneira usual. Focalizaremos agora a parte que nos interessa, a parte dipolo-quadrupolo in duzido da interação.

o limite inferior da energia de interação dipolo-quadrupolo é obtido pela substituição do segundo têrmo de $H^{\prime}$ da Eq. (5) no lado direito da Eq. (19)

$-W(R) \leq \frac{9 e^{4}}{4 R^{8}} \cdot \frac{\left\langle O\left|\left[r_{1}^{2} x_{2}-r_{2}^{2} x_{1}+\left(2 y_{1} y_{2}+2 z_{1} z_{2}-3 x_{1} x_{2}\right)\left(x_{1}-x_{2}\right)\right]^{2}\right| O>\right.}{E_{O}}$

Calculando o numerador da Eq.(20) e substituindo o va lor $E_{O}=-2 e^{2} / 2 a_{O}$ obtemos

$$
-W(R) \geq 135 \frac{e^{2} a_{0}^{7}}{R^{8}}
$$


Waals dipolo-dipolo calculada por London em têrmos da polarizabilidade elétrica dependente da frequência. Por esta razão chamou essas forças de forças de dispersões de London. Iremos agora exprimir a parte dipolo-quadrupolo da interação em têrmos da polarí zabilidade dipolar e quadrupolar.

$\mathrm{Na}$ interação dipolo-quadrupolo o călculo do numerador da Eq. (7) envolve têrmos iguais ao do cálculo da Eq. (11). Os únicos têrmos do cálculo da Eq. (7) que dão contribuição diferente de zero, no cālculo do valor esperado, são os têrmos quadrátioos de cada coordenada. Notemos também que os elementos de matriz de $x_{1}^{2}$, $y_{1}^{2}$ e $z_{1}^{2}$ são todos iguais pois o nosso estado fundamental é esferi camente simétrico. Temos então que cada um deles pode ser colocado sob a forma de $1 / 3$ do valor $\vec{r}_{1}^{2}$.

Obtemos deste modo, para a parte dipolo-quadrupolo da Eq. (7), a expressão

$-W(R)=\left(\frac{3 e^{2}}{2 R^{4}}\right)^{2} \sum_{\substack{n_{1} \neq 0 \\ n_{2} \neq 0}} \frac{|<0| r_{1}^{2}\left|n_{1}>\right|^{2}|<0| r_{2}\left|n_{2}\right|^{2}}{\left(E_{n_{1}}-E_{o_{1}}\right)+\left(E_{n_{2}}-E_{o_{2}}\right)} \ldots$

Podemos separar agora a soma dos dois têrmos indica dos no denominador da Eq. (22) usando a identidade

$\frac{1}{(a+b)}=\frac{2}{\pi} \int_{0}^{\infty} d u \frac{a b}{\left(a^{2}+u^{2}\right)\left(b^{2}+u^{2}\right)}$.

Substituindo (23) na Eq. (22) a energia de interaçäo dipolo-quadrupolo fica 


$$
\begin{gathered}
-W(R)=\left(\frac{3 e^{2}}{2 R^{4}}\right)^{2} \frac{2}{\pi} \int_{0}^{\infty} d u \sum_{n_{1} \neq 0} \frac{\left(E_{n_{1}}-E_{o_{1}}\right)\left|\left\langle o\left|r_{1}^{2}\right| n_{1}\right\rangle\right|^{2}}{\left[\left(E_{n_{1}}-E_{o_{1}}\right)^{2}+u^{2}\right]} . \\
\qquad \sum_{n_{2} \neq 0} \frac{\left(E_{n_{2}}-E_{o_{2}}\right)\left|\left\langle 0\left|\vec{r}_{2}\right| n_{2}\right\rangle\right|^{2}}{\left[\left(E_{n_{2}}-E_{o_{2}}\right)^{2}+u^{2}\right]} .
\end{gathered}
$$

No apēndice (I) mostramos que a polarizabilidade dependende da frequência è dada pela seguinte relaçäo

$\alpha(\hbar \omega)=\frac{2}{3} \sum_{n_{2}} \frac{\left(E_{n_{2}}-E_{0}\right)|<0| \vec{d}\left|n_{2}\right|^{2}}{\left(E_{n_{2}}-E_{0}\right)^{2}-(\hbar \omega)^{2}}$

Vemos então que o segundo fator do integrando presente na Eq. (24) è igual a $\alpha($ iu)/e.

O primeiro fator da Eq. (24) è igual a $3 \beta(i u)$ onde $\beta(H w)$ é a polarizabilidade quadrupolar, $2 t$

$\beta(K \omega)=\frac{2 e}{3 \sum_{n_{1} \neq 0}} \frac{\left.\left(E_{n_{1}}-E_{0}\right)|<0| r_{1}^{2}\left|n_{1}\right\rangle\right|^{2}}{\left[\left(E_{n_{1}}-E_{0}\right)^{2}-(\hbar \omega)^{2}\right]}$.

Concluimos então que a Eq. (24) pode ser escrita sob a forma $-W(R)=\frac{27 e^{2}}{4 \pi} \cdot \frac{1}{R^{8}} \int_{0}^{\infty} d u \alpha(i u) \beta(i u)$ 
12

Deste modo relacionamos a correção da energia de inte ração dipolo-quadrupolo de London com as polarizabilidades dipo lar e quadrupolar elétricas dependentes da frequência. 
CAPITULO II

AS FORÇAS DE DISPERSOES DE LONDON NA INTERAÇAO

ENTRE DOIS SISTEMAS NEUTROS

\section{1 - CONSIDERAÇOES GERAIS}

A teoria das forças de dispersões para distâncias intermediārias e grandes foram consideradas em particular por Lonquet -Higgins. Este autor preocupou-se em escrever os elementos de matriz de interação de modo fechado, sem expandỉ-la nos multipolos. A energia de interação eletrostática para dois sistemas quaisquer è dada pela expressão

$$
H^{\prime}=\int \frac{\rho_{1}\left(\vec{r}_{1}\right) \rho_{2}\left(\vec{r}_{1}\right)}{\left|\vec{r}_{1}-\vec{r}_{1}\right|} d \vec{r}_{1} d \vec{r}_{i}
$$

Quando os dois sistemas não estão muito próximos $H^{\prime}$ é relativamente fraca e pode ser tratada como uma perturbação. Nes ta situação tem-se, de fato, pequena superposição das funções de onda individuais dos sistemas, de modo que a troca de eletrons é desprezivel.

Vamos agora demonstrar que a correção de primeira ordem na energia é zero. Para isto vamos indicar a coordenada $\vec{r}_{1}^{\prime}$ que se refere ao segundo sistema, a uma origem situada no centro deste segundo sistema. Para isto basta fazer a troca $\vec{r}_{1}^{\prime} \rightarrow \vec{r}_{1}^{\prime}-\vec{R}$ onde $\vec{R} \bar{e}$ o vetor posição entre os centros dos dois sistemas. Como $\vec{R}$ 
$\bar{e}$ fixo $\rho\left(\vec{r}_{i}-\vec{R}_{1}\right.$ pode ser indicado simplesmente por $\rho\left(\vec{r}_{i}\right)$. Com isto $H^{\prime}$ será escrita

$$
H^{\prime}=\int \frac{\rho\left(\vec{r}_{1}\right) \rho\left(\vec{r}_{1}\right)}{\left|\vec{r}_{1}-\vec{r}_{1}^{\prime}+\vec{R}^{\prime}\right|} d \vec{r}_{1} d \vec{r}_{1}
$$

Desenvolvendo agora o denominador em sērie de potēn cia de $1 / R$. No caso atual em que ambos os sistemas são neutros, ve rificamos que $\mathrm{H}^{\prime}$ é dado por uma soma de produtos de multipolos do sistema: dipolo-dipolo, dipolo-quadrupolo, quadrupolo-quadrupolo, etc. O elemento de matriz de $H^{\prime}$ é então dado por uma soma de produtos dos elementos de matriz dos multipolos de cada átomo. O ele mento de matriz no estado fundamental de cada um destes multipo los é igual a zero pela composição de momentum angular $\left(\left\langle 0\left|y_{\ell m}\right| 0\right\rangle=0\right)$. Concluimos então que

$$
\left.<\mathrm{O}_{1} \mathrm{O}_{2}\left|\mathrm{H}^{\prime}\right| \mathrm{O}_{1} \mathrm{O}_{2}\right\rangle=0
$$

Isto verificamos explicitamente no capitulo I para as interações dipolo-dipolo e dipolo-quadrupolo. A correção de segun da ordem na energia foi escrita na Eq. (7) do capitulo I e a reescrevemos aqui,

$$
W(R)=\sum_{\substack{n \neq 0 \\ m \neq 0}} \frac{|<n \cdot m| H^{\prime}\left|O_{1} \cdot O_{2}>\right|^{2}}{\left(E_{n}^{(1)}-E_{o}^{(2)}\right)+\left(E_{m}^{(2)}-E_{o}^{(2)}\right)}
$$

Usando a Eq. (1) o elemento de matriz de $H^{\prime}$ torna-se

$$
\left\langle\mathrm{n} \cdot \mathrm{m}\left|\mathrm{H}^{\prime}\right| \mathrm{O}_{1} \cdot \mathrm{O}_{2}\right\rangle=\int \mathrm{d} \overrightarrow{\mathrm{r}}_{1} \mathrm{~d} \overrightarrow{\mathrm{r}}_{1} \frac{\left\langle\mathrm{n}\left|\rho_{1}\left(\overrightarrow{\mathrm{r}}_{1}\right)\right| \mathrm{o}_{1}\right\rangle\left\langle\mathrm{m}\left|\rho_{2}\left(\overrightarrow{\mathrm{r}}_{1}^{\prime}\right)\right| \mathrm{O}_{2}\right\rangle}{\left|\overrightarrow{\mathrm{r}}_{1}-\overrightarrow{\mathrm{r}}_{1}^{\prime}+\overrightarrow{\mathrm{R}}\right|}
$$


Substituindo a Eq. (5) na Eq. (4) obteremos

$$
W(R)=\sum_{\substack{n \neq 0 \\ m \neq 0}} \frac{\int d \vec{r}_{1} d \vec{r}_{1}^{\prime} \frac{\left(\rho_{1}\left(\vec{r}_{1}\right)_{o n}\right)\left(\rho_{2}\left(\vec{r}_{1}^{\prime}\right)\right.}{\left|\vec{r}_{1}-\vec{r}_{1}^{\prime}+\vec{R}^{\prime}\right|} \cdot \int \vec{r}_{2} d \vec{r}_{2}^{\prime} \frac{\left(\rho_{1}\left(\vec{r}_{2}\right)_{n o}\right)\left(\rho_{2}\left(\vec{r}_{2}^{\prime}\right)_{m o}^{\prime}\right.}{\left|\vec{r}_{2}-\vec{r}_{2}^{\prime}+\vec{R}\right|}}{\left(E_{n}-E_{o}\right)+\left(E_{m}^{(2)}-E_{o}^{(2)}\right)} .
$$

Usaremos a seguinte notação na Eq. (6), para os elementos da matriz de transição do operador densidade de carga

$$
\left(\rho(\vec{r})_{\text {on }}\right)=x_{n}(\vec{r})
$$

Usando a notação (7), e usando as coordenadas em relação a cada sistema em separado, a Eq.(6) è então escrita

$$
\begin{gathered}
W(R)=\int d \vec{r}_{1} d \vec{r}_{1}^{\prime} d \vec{r}_{2} d \vec{r}_{2}^{\prime}\left|\vec{r}_{1}-\vec{r}_{1}^{\prime}+\vec{R}\right|^{-1}\left|\vec{r}_{2}-\vec{r}_{2}^{\prime}+\vec{R}\right|^{-1} . \\
\qquad \sum_{\substack{n \neq 0 \\
m \neq 0}} \frac{x_{n}\left(\vec{r}_{1}\right) x_{m}\left(\vec{r}_{1}^{\prime}\right) x_{n}^{*}\left(\vec{r}_{2}\right) x_{m}\left(\vec{r}_{2}^{\prime}\right)}{\left.\left.(1)-E_{0}^{(I)}\right)+\left(E_{m}\right)-E_{0}^{(2)}\right)}
\end{gathered}
$$

Tal relação foi obtida por Boehm e Yaris [1] para des crever a interação entre os sistemas. Os autores utilizaram a teoria da reposta linear baseada nas técnicas de funções de Green de muitos corpos de Martim e Schwinger.

A Eq. (8) è a expressão bāsica que foi utilizada por Jacobi e Csanak [16] . Estes autores reescreveram a Eq. (8) de acordo com as considerações delineadas abaixo.

Podemos separar a coma da Eq. (8) em duas partes usando 
a identidade (23) do capitulo I. Usaremos tambēm a representação de Fourier do potencial Coulombiano (representação de Bethe),

$\frac{1}{\left|\vec{r}_{1}-\vec{r}_{1}+\vec{R}\right|}=\frac{1}{2 \pi} \int d \vec{q} \frac{e^{i \vec{q} \cdot\left(\vec{r}_{1}-\vec{r}_{1}+\vec{R}\right)}}{\vec{q}^{2}}$

Substituindo-se a identidade (23) do capitulo $I$ e a Eq. (9) na Eq. (8) obtēm-se

$$
\begin{aligned}
& W(R)=-\frac{2}{\pi} \int_{0}^{\infty} d u \frac{1}{2 \pi} \int d \vec{q} \frac{e^{i \vec{q} \cdot\left(\vec{r}_{1}-\vec{r}_{1}^{\prime}+\vec{R}\right)}}{\vec{q}^{2}} \frac{1}{2 \pi} \int \overrightarrow{d q} \cdot \frac{e^{i \vec{q} \cdot\left(\vec{r}_{2}-\vec{r}_{2}^{\prime}+\vec{R}\right)}}{\vec{q}^{2}} . \\
& \therefore d \vec{r}_{1} d \vec{r}_{1}^{\prime} d \vec{r}_{2} d \vec{r}_{2}^{\prime} \cdot \sum_{n \neq 0} \frac{x_{n}\left(\vec{r}_{1}\right) x_{n}^{*}\left(\vec{r}_{2}\right)\left(E_{n}-E_{0}^{(2)}\right)}{\left(E_{n}^{(1)}-E_{0}^{(1)}\right)^{2}+u^{2}} . \\
& \sum_{m \neq 0} \frac{x_{m}\left(\vec{r}_{i}\right) x_{m}^{*}\left(\vec{r}_{2}^{\prime}\right)\left(E_{m}^{(2)}-E_{o}^{(2)}\right)}{\left(E_{m}^{(2)}-E_{o}^{(2)}\right)^{2}+u^{2}} \text {. }
\end{aligned}
$$

Em seguida os autores acima mencionados introduzem nes sa Eq. (10) as transformadias de Fourier de $x_{n}\left(\vec{r}_{1}\right), x_{m}\left(\vec{r}_{1}^{\prime}\right), x_{n}^{*}\left(\vec{r}_{2}\right)$ e $x_{m}^{*}\left(\vec{r}_{2}^{\prime}\right)$,

$x_{n}(\vec{q})=\int \overrightarrow{d r}_{1} x_{n}\left(\vec{r}_{1}\right) e^{i \vec{g}_{1} \cdot \vec{r}_{1}}$

Substituindo a eq.(11) na Eq.(10) obtem-se 


$$
\begin{aligned}
& W(R)=\frac{-1}{2 \pi^{5}} \int_{0}^{\infty} d u \int d \vec{q} d \vec{q}^{\prime} \frac{e^{i \vec{q} \cdot \vec{k}}}{\vec{q}^{2}} \cdot \frac{e^{i \vec{q} \cdot \vec{R}}}{\vec{q}^{\prime 2}} . \\
& \sum_{n \neq 0} \frac{\left(E_{n}^{(1)}-E_{o}^{(1)}\right) x_{n}(q) x_{n}^{*}\left(-q^{\prime}\right)}{\left(E_{n}^{(1)}-E_{o}^{(1)}\right)^{2}+u^{2}} . \\
& \sum_{n \neq 0} \frac{\left\langle E_{m}^{(1)}-E_{0}^{(2)}\right) x_{m}(-\vec{q}) x_{m}^{*}\left(\vec{q}^{\prime}\right)}{\left(E_{m}^{(2)}-E_{0}\right)^{2}+u^{2}}
\end{aligned}
$$

Nesta dissertação consideramos a interação entre dois sistemas atômicos, para átomos de camadas fechadas. Vamos agora demonstrar que $x_{n}(\vec{q})$ pode ser separado em uma parte com dependência radial e outra com dependência angular. Para isto reescreve mos a Eq. (11)

$x_{n}(\vec{q})=\int e^{i \vec{q} \cdot \vec{r}} x_{n}(\vec{r}) d \vec{r}$

Vamos substituir na Eq. (13) o número quântico $n$ pela densidade $\bar{n} L M$ (n é o nūmero quântico principal e LM são números quânticos de momentum angular) para explicitar a separação das partes radial e angular. No caso do átomo de hidrogênio no estado fundamental temos

$$
\begin{aligned}
& x_{\vec{n} L M}(\vec{r})=\int \psi_{100}^{*}\left(\vec{r}^{\prime}\right) \rho\left(\vec{r}^{\prime}\right) \psi_{\vec{n} L M}\left(\vec{r}^{\prime}\right) d^{3} r^{\prime} \\
& \rho\left(\vec{r}^{\prime}\right)=e \delta\left(\vec{r}-\vec{r}^{\prime}\right)
\end{aligned}
$$


Substituindo-se (15) em (14) e integrando obtemos

$x_{\overline{n L M}}(\vec{r})=e \psi_{100}^{*}(\vec{r}) \psi_{\bar{n} L M}(\vec{r})$

Substituindo a Eq.(16) na Eq. (13) obtemos

$x_{\vec{n} L M}(\overrightarrow{\mathrm{q}})=e \int \mathrm{e}^{i \overrightarrow{\mathrm{q}} \cdot \vec{r}} \psi_{100}^{\star}(\overrightarrow{\mathrm{r}}) \psi_{\overline{\mathrm{n}} L M}(\overrightarrow{\mathrm{r}}) \mathrm{d} \overrightarrow{\mathrm{r}}$

No caso atual as funções de ondas são dadas por

$\psi_{100}(\vec{r})=R_{10}(r) Y_{00}(\theta, \phi)$
$\psi_{\bar{n} L M}(\vec{r})=R_{\bar{n} L}(r) Y_{L M}(\theta, \phi)$

Vamos usar na Eq. (17) a expansão parcial de uma onda

plana,

$e^{i \vec{q} \cdot \vec{r}}=4 \pi \sum_{\ell=0}^{\infty} i^{\ell} j_{\ell}(q r) \sum_{m=-l}^{+l} Y_{\ell m}^{\star}(\theta, \phi) Y_{\ell m}(\hat{q})$.

Nesta equação $j_{\ell}(x)$ são as funções esfēricas de Bessel e $Y_{\ell m}(\hat{q})$ são os harmônicos esféricos. Usaremos tambēm a relação de ortogonalidade

$$
\int \mathrm{Y}_{L M}(\theta, \phi) \mathrm{Y}_{\ell \mu}^{\star}(\theta, \phi) \mathrm{d} \Omega=\delta_{L \ell} \delta_{M_{\mu}}
$$

Substituindo-se as Eqs. (20), (19) e (18) na Eq. (17) ob temos 


$$
x_{\bar{n} L M}(\vec{q})=(4 \pi)^{l / 2} i^{L} e \int_{0}^{\infty} j_{L}(q R) R_{10}(r) R_{\bar{n} L}(r) r^{2} d r \cdot Y_{L M}\left(0 q^{\prime} q_{q}\right) .
$$

Podemos então escrever essa equação sob a forma

$$
x_{\bar{n} L M}(\vec{q})=x_{\bar{n} L}(q) Y_{L M}(\hat{q})
$$

onde

$$
x_{\vec{n} L}(q)=(4 \pi)^{1 / 2} i_{i}^{L} \text { e } \int_{0}^{\infty} j_{L}(q r) R_{10}(r) R_{\bar{n} L}(r) r^{2} d r
$$

Na Eq. (22) q refere-se à parte radial e $\hat{q}=\left(\theta_{q}, \phi_{q}\right)$ refere-se à parte angular. Esta relação foi obtida por Csanak Taylor [12]. Usaram tal relação para determinar o comportamento de $X_{\bar{n} L}(q)$ para pequenos e grandes valores de $q$. Substituindo-se na Eq.(12) a Eq.(22) obtém-se

$$
W(R)=\frac{-1}{2 \pi^{3}} \int_{0}^{\infty} d u \int d \vec{q} d \vec{q}^{\prime} \frac{e^{i \vec{q} \cdot \vec{R}}}{\vec{q}^{2}} \cdot \frac{e^{i \vec{q}^{\prime} \cdot \vec{R}}}{\vec{q}^{\prime 2}}
$$$$
\cdot \sum_{\bar{n} L M} \frac{\left(E_{\bar{n} L}^{(1)}-E_{O}^{(1)}\right) x_{\bar{n} L}(q) x_{L M}(\hat{q}) x_{\bar{n} L}^{*}\left(q^{\prime}\right)(-1)^{L} x_{L M}\left(q^{\prime}\right)}{\left[\left(E_{\bar{n} L}^{(1)}-E_{O}^{(1)}\right)^{2}+u^{2}\right]}
$$$$
\cdot \sum_{\bar{m} L^{\prime} M^{\prime}} \frac{\left(E_{\bar{m} L^{\prime}}^{(2)}-E_{O}^{(2)}\right) x_{\bar{m} L^{\prime}}(q)(-1)^{L^{\prime}} y_{L^{\prime} \mu}(\hat{q}) x_{\bar{m} L^{\prime}}^{*}\left(q^{\prime}\right) Y_{L^{\prime} \mu^{\prime}}\left(\hat{q}^{\prime}\right)}{\left[\left(E_{\bar{m} L}^{(2)}-E_{O}^{(2)}\right)^{2}+u^{2}\right]} .
$$ 
Vamos usar a Eq. (24) a Eq. (19) e a relação entre os es lericos harmônicos e us coeficientes de Wigner, que são dados pe1 a relação

$$
\begin{aligned}
\int d \hat{q} Y_{\ell m}(\hat{q}) Y_{L M}(\hat{q}) Y_{L^{\prime} \mu^{\prime}}(\hat{q})= & {\left[\frac{(2 L+1)\left(2 L^{\prime}+1\right)(2 \ell+1)}{4 \pi}\right]^{1 / 2} } \\
& \cdot\left(\begin{array}{lll}
\ell & L & L^{\prime} \\
0 & 0 & 0
\end{array}\right)\left(\begin{array}{lll}
L & L^{\prime} & l^{\prime} \\
M & \mu & M
\end{array}\right)
\end{aligned}
$$

Note-se que a Eq̣. (25) sō é não nula para todos os valo res de $\ell$ que satisfazem a condição triangular [15] •

Substituindo-se as Eqs. (25) e (19) na Eq. (24) obtēm-se

$$
\begin{aligned}
W(R)= & -\frac{2}{\pi^{3}} \int_{0}^{\infty} d u \int_{0}^{\infty} d q \int_{0}^{\infty} d q^{\prime} \sum_{L L ' I}\left(\begin{array}{lll}
L & L^{\prime} & I \\
0 & 0 & 0
\end{array}\right)^{2} \\
& \cdot j e(q r) j e\left(q^{\prime} r\right)\left[\frac{(2 L+1)\left(2 L^{\prime}+1\right)(2 l+1)}{4 \pi}\right] \\
& \cdot \sum_{\bar{n} \neq 0} \frac{\left(E_{\bar{n} L}^{(1)}-E_{O}^{(1)}\right) x_{\bar{n} L}(q) x_{\bar{n} L}^{*}\left(q^{\prime}\right)}{\left[\left(E_{\bar{n} L}^{(1)}-E_{O}^{(1)}\right)^{2}+u^{2}\right]} \\
& \sum_{\bar{m} \neq 0} \frac{\left(E_{\bar{m} L}^{(2)}-E_{O}^{(2)}, x_{\bar{m} L}^{(q)} x_{\bar{m} L}^{*}\left(q^{\prime}\right)\right.}{\left[\left(E_{\bar{m} L}^{(2)}-E_{O}^{(2)}\right)^{2}+u^{2}\right]}
\end{aligned}
$$

Esta é a expressão final obtida por Jacobi e Csanak [16]. Estes autores analisaram em seguida o têrmo $L=1, L^{\prime}=1$ correspon dente à parte de dipolo-dipolo induzido da interação. No prōximo parágrafo delireamos os resultados obtidos por estes autores. 
2.2 - A INTEFAÇÃO DIPOLO-DIPOLO DAS FORÇAS DE DISPERSÕES DE LONDON

Neste parágrafo delineamos o cálculo de Jacobi e Csanak [16] . O têrmo $L=1$ e $L^{\prime}=1$ da Eq. (26) è a contribuição dipo10-dipolo da energia de interação.

Csanak e Taylor [12] escreveram os primeiros têrmos da expansão de $x_{\bar{n} L}(q)$ e calcularam os coeficientes desta expansão. Jacobi e Csanak [16] utilizaram o tērmo dominante de $x_{\bar{n} l}(q)$ na Eq. (26) para calcular a energia de interação dipolo-dipolo

$x_{n I}(q)=D_{n} \frac{\alpha^{6} q}{\left(q^{2}+\alpha^{2}\right)^{3}}$

Na Eq. (27) $D_{n}$ è o elemento de matriz de transição de dipolo e a è a média do inverso dos raios dos estados orbitais (fundamental e excitado). Lassettre [17] mostrou que $\alpha$ è praticamente independente de $\mathrm{n}$. Por isso, os cālculos são feitos com $\alpha$ independente de $n$.

A substituição de $x_{n I}(q)$ na $E q$. (26) resulta na seguinte forma analitica para o têrmo dipolo-dipolo da energia de interação

$W^{(I, I)}=\frac{-3 W}{R^{6}}\left\{\left[I-e^{-\alpha R} P_{7}(\alpha R)\right]^{2}+\frac{2}{9}(\alpha R)^{6}\left[e^{-\alpha R} P_{4}(\alpha R)\right]\right\}$,

onde $\mathrm{P}_{4}(\alpha \mathrm{R})$ e $\mathrm{P}_{7}(\alpha \mathrm{R})$ são polinômios de $4 \mathrm{a}$. ordem e $7 \mathrm{a}$. ordem da- 
dos pelas seguintes expressões

$$
\begin{aligned}
& p_{4}(x)=\frac{1}{512}\left(7+7 x+3 x^{2}+\frac{2}{3} x^{3}+\frac{2}{30} x^{4}\right), \\
& p_{7}(x)=1+x+\frac{1}{2} x^{2}+\frac{1}{6} x^{3}+\frac{1}{24} x^{4}+\frac{31}{3840} x^{5}+\frac{1}{11520} x^{6}+\frac{1}{11520} x^{7}
\end{aligned}
$$

e W è a quantidade dada pela relação

$$
w=\int d \omega \alpha_{1}(i \omega) \alpha_{2}(i \omega)
$$

$\alpha(i \hbar \omega)$ é a polarizabilidade dependente da frequência ' introduzida no capitulo I.

Para grandes valores de $R$ tem-se os seguintes limites

$$
\begin{aligned}
& {\left[1-e^{-\alpha R} P_{7}(\alpha R)\right]^{2}+1} \\
& {\left[e^{-\alpha R} P_{4}(\alpha R)\right]^{2} \rightarrow 0}
\end{aligned}
$$

(1,1)

Da Eq. (28) vemos então que para $R$ grande $W(R) \therefore \therefore$ ten de ao valor $\left(-3 \int d \omega \alpha_{1}(i \omega) \alpha_{2}(i \omega) / \pi R^{6}\right)$. Este é o comportamento da energia de interação dipolo-dipolo de London, como obtido por Dal garno $[18]$.

No capitulo III estudaremos a estensäo desses resultados para o têrmo de dipolo-quadrupolo induzido da energia de inte ração $\left(L=1, L^{\prime}=2\right.$ e $\left.L=2, L^{\prime}=1\right)$. 
A INTERACÃO DIPOLO-QUADRUYOLO DAS FORÇAS DISPERSIVAS

Neste capitulo calculamos o têrmo dipolo quadrupolo da energia de interação entre dois átomos nos seus estados fundamentais.

O problema em pauta è o cálculo dos tērmos $I_{1}=1$, $L^{\prime}=$ ? e $L=2, L^{\prime}=1$ da Eq. (16) do capitulo II. Neste caso os valo res possíveis de $\ell$ são 1 e 3 .

A energia de interação dipolo-quadrupolo è então dada pela seguinte expressão

$$
\begin{aligned}
& W(R)^{2)}=\frac{-2}{\pi^{4}} \sum_{\ell=1,3}\left(\begin{array}{lll}
1 & 2 & \ell \\
0 & 0 & 0
\end{array}\right)^{2}\left[\frac{3 \cdot 5 \cdot(2 \ell+1)}{4 \pi}\right] . \\
& \text { - } \int_{0}^{\infty} d u \int_{0}^{\infty} d q \int_{0}^{\infty} d q^{\prime} j_{\ell}(q R) j_{\ell}\left(q^{\prime} R\right) . \\
& \sum_{\bar{n} \neq 0} \frac{\left(E_{\bar{n}_{1}}^{(1)}-E_{0}^{(1)}\right) x_{\bar{n}_{1}}(q) x_{n_{1}}^{\star}\left(q^{\prime}\right)}{\left(E_{\bar{n}_{1}}^{(1)}-E_{0}^{(1)}\right)^{2}+u^{2}} \\
& \sum_{\bar{m} \neq 0} \frac{\left(E_{\bar{m}_{2}}^{(2)}-E_{o}^{(2)}\right) x_{\bar{m}_{2}}^{(q)} x_{\bar{m}_{2}}^{*}\left(q^{\prime}\right)}{\left(E_{\bar{m}_{2}}^{(2)}-E_{O}^{(2)}\right)^{2}+u^{2}}
\end{aligned}
$$


Vamos substituir na equaçäo acima os dois primeiros termos da expansão de $x_{n L}(q)$ obtidas por Csanak e Taylor [12]. Estes têrmos säo dados por

$$
\begin{aligned}
& x_{n_{1}}(q)=D_{n} \frac{\alpha^{6} q}{\left(\alpha^{2}+q^{2}\right)^{3}} \\
& x_{n_{2}}(q)=Q_{n} \frac{\alpha^{8} q^{2}}{2\left(a^{2}+q^{2}\right)^{4}}
\end{aligned}
$$

Estas relações foram obtidas por Csanak e Taylor [12]. Mas foi Lassettre [17] que analisando a convergencia das expansões de $\mathrm{X}_{\mathrm{n}}(\mathrm{q})$ em sērie de Taylor que deu os primeiros passos para sua obtenção. Na Eq. (2) $D_{n}$ refere-se a transição de momento de dipolo. Na Eq. (3) $Q_{n}$ refere-se a transição de momento quadrupo10 .

Substituindo as Eqs. (2) e (3) na Eq. (1) obtemos

$$
\begin{aligned}
W(R)^{(2)}= & \frac{-2}{\pi^{4}} \sum_{\ell=1,3}\left(\begin{array}{lll}
1 & 2 & l \\
0 & 0 & 0
\end{array}\right)^{2} \frac{15(2 \ell+1)}{4 \pi} \\
& \cdot \int_{0}^{\infty} d q j_{\ell}(q R) \frac{\alpha^{6} q}{\left(\alpha^{2}+q^{2}\right)^{3}} \cdot \frac{\alpha^{8} q^{2}}{2\left(\alpha^{2}+q^{2}\right)^{4}} \cdot \\
& \cdot \int_{0}^{\infty} d q^{\prime} j_{\ell}\left(q^{\prime} R\right) \frac{\alpha^{6} q^{\prime}}{\left(\alpha^{2}+q^{\prime 2}\right)^{3}} \cdot \frac{\alpha^{8} q^{\prime 2}}{2\left(\alpha^{2}+q^{\prime 2}\right)^{4}} .
\end{aligned}
$$




$$
\begin{aligned}
& \cdot \int_{0}^{\infty} d u \sum_{n \neq 0}^{\prime} \frac{D-D \bar{n}^{*}\left(E_{n}^{(1)}-E_{0}^{(1)}\right)}{\left(E_{n}^{(1)}-E_{0}^{(1)}\right)^{2}+u^{2}} . \\
& \sum_{\bar{m}_{\neq 0}} \frac{Q_{m} Q_{m}^{*}\left(E_{m}^{(2)}-E_{O}^{(2)}\right)}{\left(E_{\frac{1}{m}}^{(2)}-E_{O}^{(2)}\right)^{2}+u^{2}}
\end{aligned}
$$

Notemos agora que a polarizabilidade de dipolo dependente da frequência é dada pela expressão do capitulo I para qual quer sistema. Para manter a normalização usada na referência [12] escrevemos

$$
\alpha(\omega)=\sum_{n} \frac{D_{n} D_{n}^{*}\left(E_{n}-E_{0}\right)}{\left(E_{n}-E_{0}\right)-\omega^{2}} \text {. }
$$

A polarizabilidade de quadrupolo dependente da frequência é dada pela expressão

$$
\beta(\omega)=\sum_{\mathrm{m}} \frac{Q_{\mathrm{m}} \Omega_{\mathrm{m}}^{*}\left(E_{n}-E_{0}\right)}{\left(E_{\mathrm{m}}-E_{0}\right)-\omega^{2}} .
$$

Usando as Eq. (6) e (5) na Eq. (4) esta se reduz a

$$
\begin{aligned}
W^{(1,2)}(R) & =\frac{-\alpha^{28}}{8 \pi^{4}} \sum_{\ell=1,3}\left(\begin{array}{lll}
1 & 2 & \ell \\
0 & 0 & 0
\end{array}\right)^{2} \frac{15(2 \ell+1)}{4 \pi} . \\
& \cdot I_{\ell}^{2} \cdot \int_{0}^{\infty} \mathrm{du} \alpha(i u) \beta(i u)
\end{aligned}
$$

onde Il é dado pela expressäo 
$I_{\ell}=\int_{0}^{\infty} d q j_{l}(q R) \cdot \frac{q^{3}}{\left(u^{2}+q^{2}\right)^{7}}$

Calculando os coeficientes de wigner em (7) para $\ell=1$ e 3 obtemos

$W(R)^{(1,2)}=\frac{-3 \alpha^{28}}{4 \pi^{5}}\left[I^{2}(R)+\frac{3}{2} I_{3}^{2}(R)\right] \int_{0}^{\infty} d u \alpha(i u) B(i u)$

Para a contribuição $\ell=1$ obtivemos de (8)

$$
I_{I}(R)=\frac{\pi e^{-\alpha R}}{2 \alpha^{10}}\left[\frac{7 \alpha R}{128}+\frac{7 \alpha^{2} R^{2}}{512}+\frac{\alpha^{3} R^{3}}{512}+\frac{\alpha^{4} R^{4}}{4608}+\frac{\alpha^{5} R^{5}}{45080}\right]
$$

Para a contribuiçäo $\ell=3$ obtivemos

$$
I_{3}(R)=\frac{7,5}{\alpha^{10}} \cdot \frac{1}{(\alpha R)^{4}}\left[1-e^{-\alpha R} P_{9}(\alpha R)\right]
$$

Nestes cálculos usamos as seguintes relações $[13,14]$

$$
\int_{0}^{\infty} \frac{\cos (a x) d x}{\left(b^{2}+x^{2}\right)^{n}}=\frac{\pi e^{-a b}}{(2 b)^{2 n-1}(n-1) !} \sum_{k=0}^{n-1} \frac{(2 n-k-2) !(2 a b)^{k}}{k !(n-k-1) !}
$$

$$
\int_{0}^{\infty} \frac{x \operatorname{sen}(a x) d x}{\left(x^{2}+b^{2}\right)^{n+1}}=\frac{\pi a e^{-a b}}{2^{2 n} n ! b^{2 n-1}} \sum_{k=0}^{n-1} \frac{(2 n-k-2) !(2 a b)^{k}}{k !(n-k-1) !}
$$




$$
\begin{aligned}
& \int_{0}^{\infty} \frac{\operatorname{sen}(a x) d x}{x\left(\beta^{2}+x^{2}\right)^{n+1}}=\frac{\pi}{2 \beta^{2 n+2}}\left[1-\frac{e^{-a \beta}}{2^{n} \cdot n !} F_{n}(a \beta)\right] \\
& F_{0}(Z)=1, F_{1}(Z)=Z+2, \ldots, F_{n}(z)=(z+2 n) F_{n-1}(Z)-Z F_{n-1}^{\prime}
\end{aligned}
$$

$P_{g}(\alpha R)$ è um polinômio de nono grau. Este polinōmio é dado por

$\mathrm{Pg}(\alpha \mathrm{R})^{2}=1+\alpha \mathrm{R}+\frac{1}{46080}\left[23040(\alpha \mathrm{R})^{2}+7680(\alpha \mathrm{R})^{3}+1920(\alpha \mathrm{R})^{4}+\right.$ $\left.+\frac{887}{6}(\alpha R)^{5}+64(\alpha R)^{6}+9(\alpha R)^{7}+\frac{49}{48}(\alpha R)^{8}+\frac{2}{15}(\alpha R)^{7}\right]$

Para grandes valores de $R$ tem-se de (10) e (13) os seguintes limites

$I_{1}(R) \rightarrow 0$

$I_{3}(R)+\frac{7,5}{\alpha^{14}} \cdot \frac{1}{R 4}$

De (9) vemos entäo que para $\mathrm{R}$ grande $\mathrm{W}(\mathrm{R})^{(1,2)}$ tende ao valor $\left[-/ \pi^{3} \int_{0}^{\infty} \mathrm{du} \alpha(i u) \beta(i u)\right] / R^{\beta}$.

Este é o comportamento da energia de interação dipolo -quadrupolo que obtivemos na Eq. (27) no capitulo I.

o cálculo da energia de interaçäo dipolo-quadrupolo é de grande importāncia em cálculos mais precisos de interações a 
grandes distäncias e à distâncias intermediárias. Essa energia de interação é útil ha interpretação das equações de estado do hidro gênio sólido comprimido [19].

Lassettre [17] sugeriu a aplicação destes cálculos pạ ra calcular-se o potencial eletrostático dos átomos. Estas expres sões foram usadas com sucesso [12] na determinação do potencial de polarização de espalhamento de elétrons por átomos. 
CONTRIBUIÇðES ORIGINAIS DESTE TRABALHO

1. Càlculo dos limites superior e inferior da parte dipolo-quadrupolo da energia de inte ração entre dois sistemas neutros.

2. Cálculo da parte dipolo-quadrupolo da ener gia de interação entre dois sistemas neutros. 


\section{CONCLUS ̃̃O}

Esta dissertação estuda a energia de interação de Van der Wals entre dois sistemas neutros. Esta forma de energia foi formulada por London na situação de grandes distāncias segurando o têrmo correspondente à parte dipolo-dipolo induzido da interação, que é o têrmo dominante a grandes distâncias. Em sequida des tacamos o trabalho de Longuet-Higgins crue escreveu os elementos de matriz de interação de modo fechado, sem expandi-la nos multipolos. O potencial de interação é escrito em têrmos dos elementos de transição da densidade elétrica. A transformada de Fourier des ses elementos de matriz foi analisada por Lassettre. Csanak e Tay lor notaram que as considerações de Lassettre poderia ser utilizadas para calcular as expansões destas transformadas. Jacobi e Csanak usaram estas expansões para o cálculo da parte correspon dente à parte dipolo-dipolo da interação a distância intermediā ria e grande.

Nesta dissertação estudamos a parte seguinte, dipoloquadrupolo induzido da interação.

Analisamos inicialmente o caso de grandes distäncias, seguindo a teoria estabelecida por London para a parte dipolo-dipolo da interação. A seguir estudamos o caso de distâncias intermediārias extendendo deste modo os resultados de dipolo-dipolo in duzido obtidos por Jacobi e Csanak. 


\section{APENDICE I}

\section{A POLARIZABILIDADE}

A polarizabilidade $\alpha$ de um àtomo è definida em têrmos do campo elētrico local no àtomo

$$
\vec{d}_{\text {ind }}=\alpha \vec{E}
$$

onde $\overrightarrow{\mathrm{d}}_{\text {ind }} \mathrm{e}$ o momento de dipolo elétrico induzido no sistema. 0 dipolo elētrico do sistema no estado caracterizado por $|\psi(t)\rangle \quad \bar{e}$ dado pelo valor médio do operador dipolo-elētrico, $\langle\psi(t)|\bar{d}| \psi(t)\rangle$. Vamos calcular a polarizabilidade do sistema quando a perturbação é devida a um campo elétrico oscilante e orientado no sentido do eixo $z$. A energia de interação é então dada pela relação

$H^{\prime}(t)=-d_{z} E=-d_{z}\left(E_{O} e^{i \omega t}+E_{O}^{*} e^{-i \omega t}\right) e^{\varepsilon t}$

Nessa equação jā introduzimos o fator de ligação adiabätica em $-\infty, \exp (\varepsilon t)$.

Atē primeira ordem de perturbaçäo o vetor de estado do sistema è dado por

$$
|\psi(t)\rangle=\sum_{n}|n\rangle\left(\delta_{n a}+\frac{1}{i \hbar} \int_{t_{0}}^{t} H_{n a}^{\prime}\left(t^{\prime}\right) e^{i\left(\omega_{n}-\omega_{a}\right) t^{\prime}} d t^{\prime}\right) e^{-i \omega_{n} t} .
$$

$$
\text { Usando as Eqs. (2) e (3) podemos calcular o valor médio }
$$

de $\mathrm{d}_{\mathbf{z}}$ 
$\left\langle\psi(t)\left|d_{z}\right| \psi(t)\right\rangle=\int \psi^{*}(t) d_{z} \psi(t) d \tau$

Usando (3) em (4) obtemos

$\left\langle\psi(t)\left|d_{z}\right| \psi(t)\right\rangle=\left\langle a\left|d_{z}\right| a\right\rangle+$

$$
\begin{aligned}
& \left.+\frac{1}{i \hbar} \sum_{n^{\prime}}<a\left|d_{z}\right| n\right\rangle \int_{t=-\infty}^{t^{\prime}} H_{n a}^{\prime}\left(t^{\prime}\right) e^{i\left(\omega_{n}-\omega_{a}\right) t^{\prime}} d t^{\prime} e^{i\left(\omega_{j}-\omega_{n}\right) t}- \\
& -\frac{1}{i \hbar} \sum_{n^{\prime}}\left\langle n\left|d_{z}\right| a\right\rangle \int_{t_{0}=-\infty}^{t^{\prime}} H_{a n}^{\prime}\left(t^{\prime}\right) e^{-i\left(\omega_{n}-\omega_{a}\right) t^{\prime}} d t^{\prime} e^{-i\left(\omega_{a}-\omega_{n}\right) t}
\end{aligned}
$$

Calculando as integrais em (5) obtemos

$$
\begin{aligned}
\int_{-\infty}^{t} H_{n a}^{\prime}\left(t^{\prime}\right) e^{i\left(\omega_{n}-\omega_{a}\right) t^{\prime}} d t^{\prime}=-\left\langle n\left|d_{z}\right| a>E_{0}\right. & \frac{e^{\left[i \omega+\varepsilon+i\left(\omega_{n}-\omega_{a}\right) t\right.}}{\left[i \omega+\varepsilon+i\left(\omega_{n}-\omega_{a}\right)\right]}+ \\
& +E_{0}^{*} \frac{e^{\left[-i \omega+\varepsilon+i\left(\omega_{n}-\omega_{a}\right)\right] t}}{\left[-i \omega+\varepsilon+i\left(\omega_{n}-\omega_{a}\right)\right]} .
\end{aligned}
$$

Substituindo-se a Eq. (6) na Eq. (5) obtemos

$\left\langle\psi(t)\left|d_{z}\right| \psi(t)\right\rangle=\left\langle a\left|d_{z}\right| a\right\rangle+\sum_{n}\left|\left\langle a\left|d_{z}\right| n\right\rangle\right|^{2} \frac{1}{\hbar}$.

$$
\left\{E_{0}\left(\frac{e^{(i \omega+\varepsilon) t}}{\left(\omega_{n}-\omega_{a}\right)+\omega-i \varepsilon}+\frac{e^{(i \omega+\varepsilon) t}}{\left(\omega_{n}-\omega_{a}\right) \cdot \omega+i \varepsilon}\right)+\begin{array}{l}
\text { Complexo } \\
\text { Conjugado }
\end{array}\right\} \cdot(7)
$$


Desenvolvendo essa equação obtemos, depois de fazer $\varepsilon \rightarrow 0$

$\left\langle\psi(t)\left|d_{z}\right| \psi(t)\right\rangle=\left\langle a\left|d_{z}\right| a\right\rangle+E \frac{E}{n} \sum_{n}\left|\left\langle a\left|d_{z}\right| n\right\rangle\right|^{2} \frac{2\left(\omega_{n}-\omega_{a}\right)}{\left(\left(\omega_{n}-\omega_{a}\right)^{2}-\omega^{2}\right)}$

O primeiro têrmo no lado direito da Eq. (8) é o dipolo permanente e o segundo térmo é o dipolo induzido. Temos então $a_{\text {ind }}=\alpha(\omega)$ E onde $\alpha(\omega)$ è dada pela relaçäo

$\alpha(\omega)=\frac{2}{\hbar} \sum_{n} \frac{\left(\omega_{n}-\omega_{a}\right)|<a| d_{z}|n>|^{2}}{\left[\left(\omega_{n}-\omega_{a}\right)^{2}-\omega^{2}\right]}$

Para um sistema no estado fundamental $|a>=| 0>$, especifi camente simētrico, teremos um resultado anālogo tanto para o campo elétrico orientado no sentido de $x$ como no sentido de $y$. Portanto podemos escrever

$$
\alpha=\frac{2}{3} n_{n} \frac{\left|\left\langle u\left|\vec{a}_{z}\right| 0\right\rangle\right|^{2}\left(E_{n}-E_{0}\right)}{\left(E_{u}-E_{0}\right)^{2}-(h \omega)^{2}}
$$


REFERENCIAS BIBLIOGRAFICAS

[1] - D.Van der Waals, Die Kontinuital de gas formigenund flulssigen Zustandes (Amsterdam, 1981).

[2] - L. Boltzman, Wied. Ann. 24, 37 (1885).

[3] - C. Maxwe11, Phil. Mag. (4) 34, 129, 185 (1868).

[4] - M. Reinganum, Ann. d. Physik 10, 334 (1903); 38, 649 (1912).

[5] - W.H. Keeson, Physik Zeits 22, 129 (1921).

[6] - P. Debye, Physk Zeits 21, 178 (1920).

[7] - H. Falkenhagen, Physik Zeits 23,87 (1922).

[8] - (a) R. Eisenschitz and F. London, Zeits F. Physik 60, 491 (1930); (b) F. London, Zeits F. Physik Chemie (B) 11, 222 (1930).

[9] - F. London, Zeits F. Physik 63, 245 (1930).

[10]-H.C. Longuet-Higgins, Proc. Roy. Soc. A 235 (1956) 537.

[11] - R. Boehm e R. Yaris, J.Chem. Phys. 55 (1971) 2620.

[12] - G. Csanak; A.S. Taylor, Physical Review A, 6,5 (1972).

[13] - I.S. Gradsteyn e I.M. Ryzhik, Table of Integras series and Products - Academic Press (1965).

[14] - Handbook of Mathematical Functions (1964).

[15] - A.R. Edmonds, Angular Momentum in Quantum Mechanics (1957). 
35

[16] - Jacobi e Csanak, Chem. Phys. Letters 30, 3 (1975)

[17] - E.N. Lassettre, J. Chem. Phys. 43, 12(1965) (4479).

[18] - A. Dalgarno, Advan. Chem. Phys. 12(1967) 143.

[19] - M. Ross, J. Chem. Phys., 60 (1974) 3634.

[20] - L. Pauling e L.B. Wilson, Jr. - Introduction to Quantum Mechanics, McGraw-Hill, New York (1935). 